\title{
Microwave Encoders for Chipless RFID and Angular Velocity Sensors based on S-Shaped Split Ring Resonators (S-SRRs)
}

\author{
Cristian Herrojo, Student Member, IEEE, Javier Mata-Contreras, Ferran Paredes, Member, IEEE, and \\ Ferran Martín, Fellow, IEEE
}

\begin{abstract}
In this paper, it is demonstrated that a chain of Sshaped split ring resonators (S-SRRs) etched on a dielectric substrate can modulate the amplitude of a carrier signal injected to a transmission line (a coplanar waveguide -CPW). To this end, the S-SRR chain must be transversally displaced above the CPW, in close proximity to it. By this means, the transmission coefficient of the line is modulated by the time-varying electromagnetic (inductive) coupling between the line and the $S$ SRRs of the chain, related to their relative motion. Based on this principle, two different applications can be envisaged: (i) angular velocity sensors, and (ii) near-field chipless radiofrequency identification (chipless-RFID) tags. In the former application, the S-SRR chain is circularly shaped and the S-SRRs are distributed uniformly along the perimeter of the rotor, at equidistant positions. By this means, the amplitude modulated signal generated by rotor motion exhibits envelope peaks whose distance is related to the angular velocity of the rotor. In the use of S-SRRs as microwave encoders for chipless RFID tags, not all the S-SRRs of the chain are present. Their presence or absence at the predefined (equidistant) positions is related to the logic state ' 1 ' or ' 0 '. Tag reading is sequential and it is achieved through tag motion (at constant velocity) above the reader, a CPW transmission line fed by a carrier signal. The identification (ID) code is contained in the envelope function of the resulting amplitude modulated signal, which can be obtained by means of an envelope detector. With the proposed approach, a high number of pulses in angular velocity sensors can be achieved (with direct impact on angle resolution and sensitivity to changes in instantaneous rotation speed). Moreover, chipless-RFID tags with unprecedented number of bits can be obtained. The proposed angular velocity sensors can be useful in space environments, whereas the chipless-RFID systems based on the proposed tags are useful in applications where reading range can be sacrificed in favor of high data capacity (large number of bits), e.g., security and authentication.
\end{abstract}

Index Terms-Angular velocity sensors, chipless RFID, coplanar waveguide, $s$-shaped split ring resonators (S-SRRs).

\section{INTRODUCTION}

$\mathrm{T}$ ransmission lines loaded with electrically small resonators have been used in many different microwave applications

This work was supported by MINECO-Spain (projects TEC2013-40600-R and RTC-2014-2550-7), Generalitat de Catalunya (project 2014SGR-157), ICREA, and by FEDER funds. C. Herrojo acknowledges MINECO for supporting his research activity through the FPI grant BES-2014-068164.

The authors are with GEMMA/CIMITEC, Departament d'Enginyeria Electrònica, Universitat Autònoma de Barcelona, 08193 Bellaterra, Spain. Email: Ferran.Martin@uab.es
$[1,2]$. In particular, it has been demonstrated that split ring resonator (SRR) loaded lines [3] and complementary split ring resonator (CSRR) loaded lines [4,5] are useful for the implementation of one dimensional (1D) metamaterials. By combining SRRs with shunt inductors and CSRRs with series capacitors, left-handed lines exhibiting antiparallel phase and group velocities within a certain frequency band have been reported [3,5]. The controllability of the dispersion relation and characteristic impedance in these artificial lines has opened the door to the design of microwave devices with superior performance or with novel functionalities, on the basis of impedance and dispersion engineering [1,2].

In other applications, where a transmission line is simply loaded with resonant elements (SRR, CSRR, or other electrically small resonators), the resonance phenomenon, rather than dispersion and impedance engineering, is exploited [1]. Such resonator-loaded lines exhibit stopband functionality useful in many different microwave applications, such as blocking spurious signals in microwave circuits [6], multiband dipole and monopole antennas based on the concept of trap antennas [7,8], microwave sensors [9] and chipless-RFID tags [10].

Concerning sensors implemented by means of resonator loaded lines, various approaches have been presented. Most of such sensors are based on the variation of resonance frequency caused by the variable to be measured (measurand) [11-17]. Other sensors implemented by means of resonator loaded lines are based on symmetry properties, including coupling modulation sensors [9,18-24] and sensors based on frequency splitting [25-28]. In coupling modulation sensors, a transmission line is symmetrically loaded with a symmetric resonator. The key aspect for sensing purposes is that the resonant element exhibits a symmetry plane acting as electric wall (at the fundamental resonance, the one of interest), provided the transmission line exhibits a magnetic wall at its axial symmetry plane (as occurs in most usual transmission lines, such as microstrip, CPW, etc.). If symmetry is preserved, the resonator is not excited due to perfect cancellation of electric and magnetic fields on the resonator area. However, if symmetry is broken, e.g., by the effects of a spatial variable, or by an asymmetric dielectric load, then the resonator is excited and a notch in the transmission coefficient appears. Moreover, the magnitude (depth) of such notch is related to the level of asymmetry, and can be used as output 
variable in these coupling modulation sensors. In frequency splitting sensors, a transmission line is symmetrically loaded with a pair of identical (but not necessarily symmetric) resonant elements. Under these conditions, the structure exhibits a single transmission zero (notch) in the vicinity of the fundamental resonance frequency of the loading elements. Conversely, when symmetry is broken, frequency splitting appears, and the separation between the two notches gives a measure of the level of asymmetry. Both, coupling modulation and frequency splitting sensors are robust in front of cross sensitivities caused by environmental factors such as temperature and moisture drifts. The reason is that symmetry is invariant under changing ambient conditions.

There is another type of sensors where the transmission coefficient of a transmission line is modulated by the effects of a movable resonant element, or set of resonant elements, in close proximity to the line. In [22], angular velocity sensors based on electric LC (ELC) resonators were reported. In that work, the transmission line is a CPW and the ELC resonator is axial to the rotor. Since the ELC resonator is a bisymmetric resonator exhibiting orthogonal electric and magnetic walls at the fundamental resonance, by rotating the ELC resonator in close proximity to the CPW transmission line, line-toresonator coupling is modulated and, consequently, the transmission coefficient varies between a maximum and a minimum value twice per cycle. By injecting a harmonic signal (carrier) tuned to the resonance frequency of the ELC resonators, the amplitude is modulated at the output, and the distance between adjacent maxima, or minima, in the envelope function corresponds to half the period of the rotor. Variations of the angular velocity sensor proposed in [22] can be found in $[23,24]$.

In the previous angular velocity sensors, also useful as angular displacement sensors, two pulses per cycle are generated. This prevents from the measurement of instantaneous angular velocities that can vary during a cycle. To solve this problem, the edge configuration was presented in [29], where the rotor was equipped with a circular chain of SRRs etched along its external perimeter. By situating the stator, a CPW transmission line, just below the SRR chain, the transmission coefficient of the line is modulated at the rhythm of the SRRs passing on top of the CPW axis. Since the number of SRRs of the chain can be made very large (300 in [29]), it follows that the number of pulses of the resulting envelope function is also large. Therefore, the measurement of quasiinstantaneous angular velocities is possible. In a recent work [30], an angular velocity sensor exhibiting 1200 pulses per cycle, based on a double chain of SRRs and with optimized carrier frequency, was reported.

Concerning chipless-RFID tags, there are three main approaches for their implementation: (i) time domain based tags, (ii) frequency domain based tags and (iii) hybrid tags. Time domain based tags are realized by loading a transmission line with scatterers, and the interrogation signal is a pulse injected to it [31-40]. The ID code is inferred from the echoes of that signal. In frequency domain based tags, a transmission line is loaded with as many resonators (each tuned to a different frequency) as number of bits (retransmission based tags) $[41,42]$. An alternative are the backscattered based tags [43-49]. The interrogation signal in both cases is a multifrequency signal that must be swept across the spectral bandwidth occupied by the resonant elements. The ID code is obtained from the singularities present in the amplitude (notches), phase or time delay response. The number of bits in retransmission based tags has been increased by using resonators able to provide more than two states. Such states are obtained from the relative orientation between the resonator and the line $[10,50,51]$, providing different notch depth. However, by this means, the number of bits achievable is still far from those of chipped RFID tags. Hybrid tags [5256] are multi-domain tags where more than one domain (e.g. time, frequency, phase, polarization, etc.) are used simultaneously in order to achieve more than one bit of information per resonant element. Examples of hybrid tags include encoders based on frequency position and polarization diversity [54], and encoders where frequency domain is combined with phase deviation [53], among others.

In this paper, chains of S-SRRs are used as microwave encoders for two different applications: angular velocity measurements and near-field chipless RFID tags. The principle, identical in both applications, is explained in Section II. Section III is focused on the design of the S-SRR chain and the S-SRR-loaded line, necessary for tag reading and for the measurement of angular velocities. The validation of the two considered applications is reported in Section IV. In Section V, the use of S-SRRs as resonant elements is justified. Finally, the main conclusions are highlighted in Section VI.

\section{PhYSICAL PRINCIPLE}

The principle of the proposed devices, first reported in [29], is the modulation of the transmission coefficient of a transmission line produced by the motion of a chain of resonant elements in close proximity to the line. The proximity is necessary since the above-cited modulation in the transmission coefficient is achieved by the time-varying electromagnetic coupling between the line and the resonant elements. As the resonator chain moves with regard to the line, such coupling varies and, consequently, the transmission coefficient, whose magnitude decreases with the coupling level, also varies.

For angular velocity measurements, the chain must be circularly-shaped and located in the external perimeter of the rotor. In this application the resonant elements must be equidistant and distributed uniformly along the rotor perimeter. If a transmission line (active part of the stator) is located in close proximity to the chain, and it is fed by a harmonic signal tuned to a certain frequency close to the resonance frequency of the resonators, rotor motion is expected to modulate the amplitude of the signal at the output port of the line (due to the modulation of the transmission coefficient). Obviously, the time distance between adjacent peaks (or valleys) in the envelope function is intimately related to the rotation speed, and hence such angular velocity can be inferred from the envelope function. Such function can 
be obtained by means of an isolator and an envelope detector (composed of a Schottky diode and a low pass filter). The isolator can be implemented by means of a circulator and it is necessary to protect the transmission line against mismatching reflections from the diode (a highly nonlinear device). The working principle for this application is illustrated in Fig. 1. In $[29,30]$, angular velocity sensors based on this principle and implemented by means of split ring resonators (SRRs) were considered. In this paper, we use S-shaped SRRs (S-SRRs), which are electrically small and are useful not only for angular velocity measurement, but also for the implementation of chipless RFID tags with high data density (advantages and disadvantages of S-SRRs as compared to SRRs are pointed out in the discussion).

For chipless RFID systems, the tags are a set of aligned resonant elements printed on a dielectric substrate (typically a flexible substrate, including organic substrates, such as paper, plastics, or liquid crystal polymers). However, the resonators are not necessarily uniformly distributed. For this application, equidistant positions for the resonant elements of the tag, each one representing a bit of information, are predefined. The logic state ' 1 ' is associated to the presence of resonant element, whereas the absence of resonator at the predefined positions corresponds to the logic ' 0 ' state. Tag reading proceeds similarly to the measurement of angular velocities (see Fig. 2). The tag must be displaced on top of a transmission line fed by a harmonic signal (tuned to a frequency close to the resonance frequency of the tag resonators). By this means, the amplitude of the feeding signal is modulated, and the ID code of the tag is contained in the envelope function of the amplitude modulated signal present at the output port of the transmission line. The necessary stages to obtain such envelope function are identical to those needed in angular velocity measurements (specified in the previous paragraph).

\section{DESIGN OF THE S-SRR CHAIN AND S-SRR-LOADED CPW TRANSMISSION LINE}

In this paper, the resonant elements of the chain (for both the rotor and the chipless tags) are S-shaped split ring resonators (S-SRRs) [57-59]. The transmission line, acting as stator (for angular velocity measurements) or as active part of the reader (for chipless RFID systems), is a coplanar waveguide (CPW) transmission line. S-SRRs are electrically small particles that can be excited by the counter magnetic field lines generated in the slots of the CPW transmission line when such particles are aligned and oriented as depicted in Fig. 3(a). For chipless RFID tags, the small electrical size of S-SRRs provides a small size for the tag. For angular velocity measurements, a large number of pulses (a figure of merit) in a certain rotor perimeter can be achieved if the particles occupy a small size. For these two reasons, S-SRRs are the resonators considered in this paper. Note that in $[29,30]$ rectangular shaped SRRs were used for angular velocity measurements (the stator was a CPW transmission line, as well). With the considered shape factor of the resonant

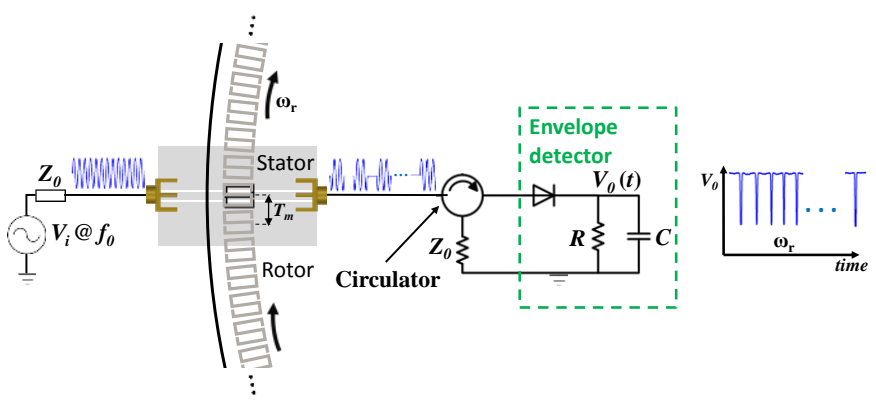

Fig. 1. Illustration of the working principle for angular velocity measurements based on the amplitude modulation of a harmonic signal produced by rotor motion.

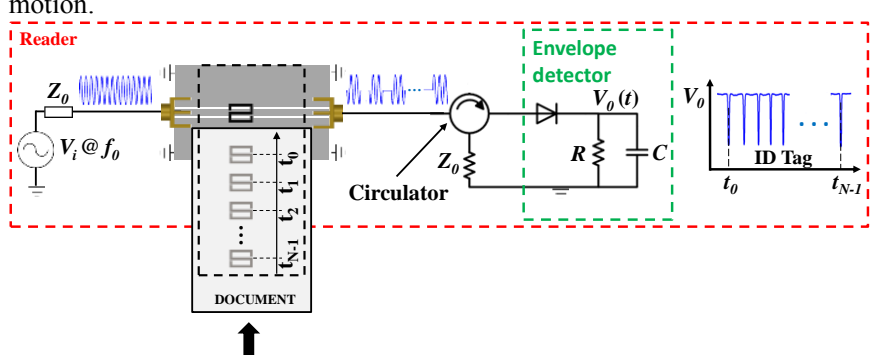

Fig. 2. Illustration of the working principle for tag reading in a chipless RFID system based on near-field coupling and sequential bit reading.

elements, the distance between adjacent resonant elements was very small, and a large number of pulses was achieved.

For both applications (angular velocity sensors and chipless RFID tags), a small distance between adjacent predefined resonator positions (either with or without S-SRRs) is very convenient. Such small separation favors compact size in chipless tags and large number of resonators (and hence pulses) in rotors. However, by tiny spacing adjacent resonators, inter-resonator coupling and simultaneous coupling between the line and several S-SRRs of the chain are favored. These effects should be avoided in order to prevent the appearance of multiple transmission zeros located at positions difficult to predict a priori. The solution to these limiting aspects related to small inter-resonator separation is to etch an identical S-SRR in the back substrate side of the CPW transmission line, but oppositely oriented [see Fig. 3(b)]. When the relative position between the S-SRR of the line and the S-SRR chain corresponds to a perfectly aligned (vertically) pair of S-SRRs (the one of the line with one of the S-SRRs of the chain), both vertically aligned S-SRRs can be seen as a single resonant element (broad-side coupled S-SRR or BC-SSRR). Since the fundamental resonance frequency of the BCS-SRR, $f_{0}$, is smaller than the one of the individual (uncoupled) resonators, it follows that by setting the frequency of the feeding signal (carrier frequency, $f_{\mathrm{c}}$ ) of the CPW transmission line (necessary for tag reading or for angular velocity measurements, as mentioned before) to $f_{\mathrm{c}}=f_{0}$, or close to it, then the above cited cross couplings are avoided. Such strategy was pointed out in [29] in reference to angular velocity measurements based on pairs of SRRs. The cross sectional view of the S-SRR chain and the S-SRR-loaded CPW, separated by the air gap, can be seen in Fig. 3(c). 
(a)

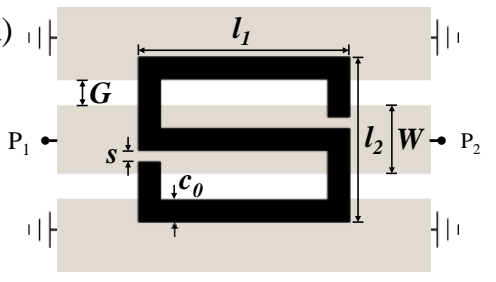

(b)

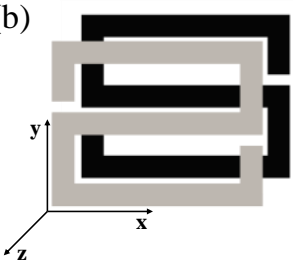

(c)

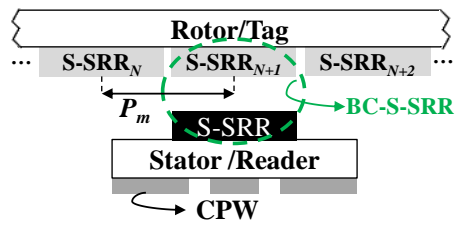

Fig. 3. Square S-shaped SRR coupled to a CPW transmission line (a) 3D view of the broadside coupled S-SRR (BC-S-SRR) (b), and cross sectional view of the stator/reader and rotor/tag (c).
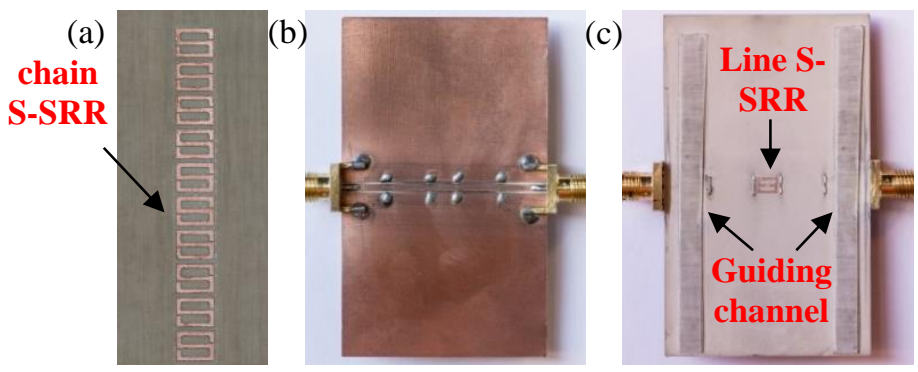

Fig. 4. Photograph of the fabricated 10-S-SRR chain (a) and top (b) and bottom (c) views of the S-SRR-loaded CPW transmission line. Dimensions are, in ref. to Fig. 2(a) and Fig. 2(b), (in mm) $W=1.2$ and $G=0.48, l_{l}=3.8$, $l_{2}=2.96, c_{0}=0.4, s=0.2$, and $P_{m}=3.5$. CPW transmission line and tag are separated by a $0.25 \mathrm{~mm}$ air gap.

The considered substrate for the implementation of the SSRR chains is the Rogers RO4003C with thickness $h=203 \mu \mathrm{m}$ and dielectric constant $\varepsilon_{r}=3.55$. Since this substrate is very narrow, and hence flexible, it has been attached to a rigid substrate $F R 4$ (with thickness $h=1.6 \mathrm{~mm}$ and dielectric constant $\left.\varepsilon_{r}=4.7\right)$. By these means, mechanical stability, necessary for tag reading and angular velocity measurements, is ensured. The CPW transmission line and the S-SRR of the reader/stator have been etched on opposite sides of a Rogers RO3010 substrate with thickness $h=635 \mu \mathrm{m}$ and dielectric constant $\varepsilon_{r}=10.2$. The size of the S-SRRs has been optimized in order to obtain a resonance frequency for the broadside coupled counterpart (BC-S-SRR) of $f_{0}=4 \mathrm{GHz}$. For characterization purposes, we have fabricated a linear chain of 10 S-SRRs [see Fig. 4(a)]. The top and bottom views of the SSRR-loaded CPW transmission line can be seen in Figs. 4(b) and (c), respectively. Note that a guiding channel has been added to the bottom side of the CPW in order to guide, with the necessary alignment, the S-SRR chain over the S-SRR of the line. We have measured the response of the structure for different positions of the S-SRR chain in the vicinity of the reference position (corresponding to a perfect alignment between the S-SRR of the line and one of the S-SRRs of the chain). The results are depicted in Fig. 5. When the tag is not situated in the reference position, the attenuation at $f_{0}$ severely

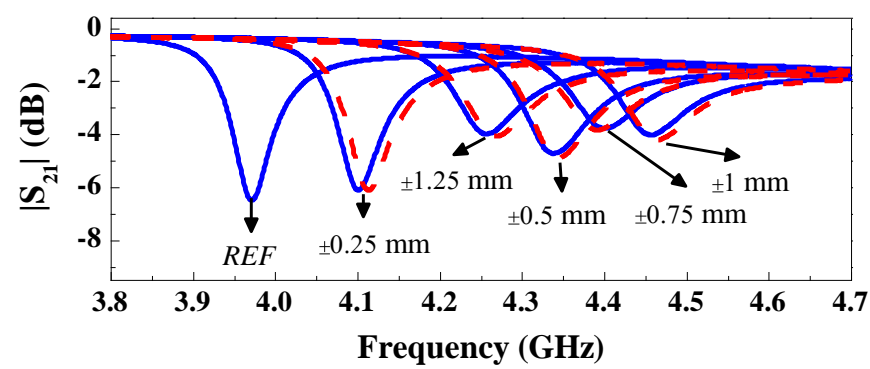

Fig. 5. Measured frequency response of the S-SRR-chain/CPW for different relative positions of the S-SRR chain in the vicinity of the reference position.

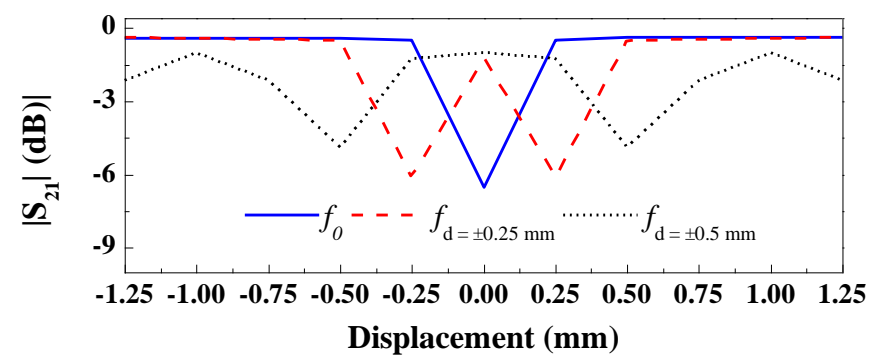

Fig. 6. Measured attenuation as a function of the relative displacement between the line and the chain at the indicated frequencies.

decreases. This can be appreciated in Fig. 6, where attenuation at different frequencies, as a function of the relative displacement between the line and the chain, is also depicted. It can be appreciated that for frequencies larger than $f_{0}$ (i.e., $f_{\mathrm{d}= \pm 0.25 \mathrm{~mm}}$ and $f_{\mathrm{d}}= \pm 0.5 \mathrm{~mm}$, corresponding to the notch frequencies for the indicated relative displacements), two attenuation peaks per chain period, rather than one, appear. The reason is that for carrier frequencies slightly larger than $f_{0}$ there are two equivalent positions (one for positive displacement and the other one for negative displacement) where the transmission coefficient is a minimum. In practice, it is difficult to perfectly set the carrier frequency to $f_{c}=f_{0}$. The reason is that it is not easy to accurately control the air gap separation between the line and the S-SRR chain. Therefore, a convenient strategy is to set $f_{c}$ above $f_{0}$, accepting that two attenuation peaks (rather than one) per chain S-SRR cross above the S-SRR of the line will appear. Through this approach, some tolerance in the air gap can be assumed. Note that by increasing the air gap distance, the effect is an overall shift of the responses to larger frequencies, as illustrated in Fig. 7. If the carrier frequency is chosen as $f_{c}=f_{0}$, and the gap increases, it may give rise to reading errors or false angular velocity measurements.

\section{APPLICATIONS}

In this section, specific S-SRR chains with identical S-SRRs as those considered in the previous section and etched in the same substrate are used for two different applications: chipless RFID systems and angular velocity measurements. For angular velocity measurements, the chain must be circularly shaped along the perimeter of the rotor, and the S-SRRs must be located at periodic positions. Since the number of resonant elements along the perimeter of the rotor is known, it follows that the angular velocity is given by the time distance between 


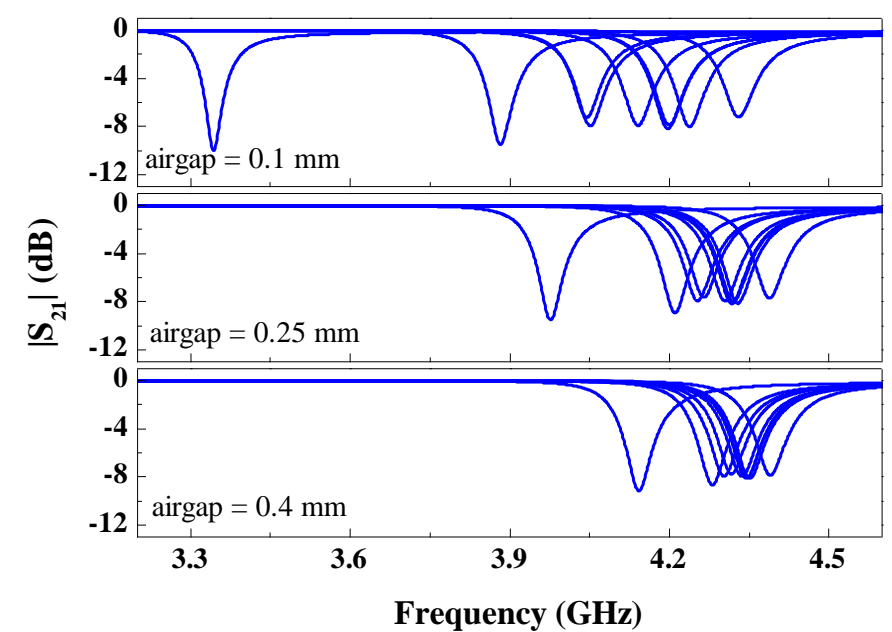

Fig. 7. Transmission coefficient, inferred from electromagnetic simulation using Keysight Momentum, for different relative displacements between the SSRR chain and the CPW, parameterized by the air gap separation.

adjacent peaks in the envelope function $\left(T_{m}\right)$, provided a single notch per S-SRR cross, above the S-SRR of the line, results. If a pair of notches is visible, typically these two notches are very close one each other as compared to the time distance between pairs of adjacent notches. Therefore, in this later case $T_{m}$ is the time distance between pairs of adjacent notches (corresponding each pair of notches to a different S-SRR cross above the S-SRR of the line). By measuring $T_{m}$ it follows that the angular velocity is given [29]

$$
\omega_{r}=\frac{d \theta}{d t}=\lim _{\Delta t \rightarrow 0} \frac{\Delta \theta}{\Delta t}=\frac{\theta_{m}}{T_{m}}=\frac{2 \pi / P P R}{T_{m}}
$$

where $\theta$ is the angular position, $t$ is time, $\theta_{m}$ is the angular period and $P P R$ is equal to the number of resonant elements in the rotor.

The photograph of the experimental setup for angular velocity measurements can be seen in Fig. 8. The circulator employed to implement the isolator is the ATM ATC4-8. The envelope detector uses the Avago HSMS-2860 Schottky diode and the N2795A Active probe (which acts as lowpass filter with $R=1$ $\mathrm{M} \Omega$ and $C=1 \mathrm{pF}$ ), connected to an oscilloscope (the Agilent $M S O-X-3104 A$ ) in order to visualize the envelope function. The carrier signal is generated by means of the Agilent E4438C function generator, whereas the displacement and velocity of the rotor is controlled by the STM 23Q-3AN stepper motor. The S-SRR loaded line has been re-designed and re-fabricated since the guiding system is not necessary [see Fig. 9(a) and (b)]. Nevertheless, it is important to vertically align the S-SRR of the line (stator) with those of the rotor, and this is achieved through stator motion in three dimensions, allowable by the experimental setup. Vertical motion is necessary to properly adjust the gap distance to the required value, i.e., $0.25 \mathrm{~mm}$, or as much close as possible to it.

For chipless RFID tags, given a number of bits (and hence resonator positions), the S-SRR chain is typically linear.

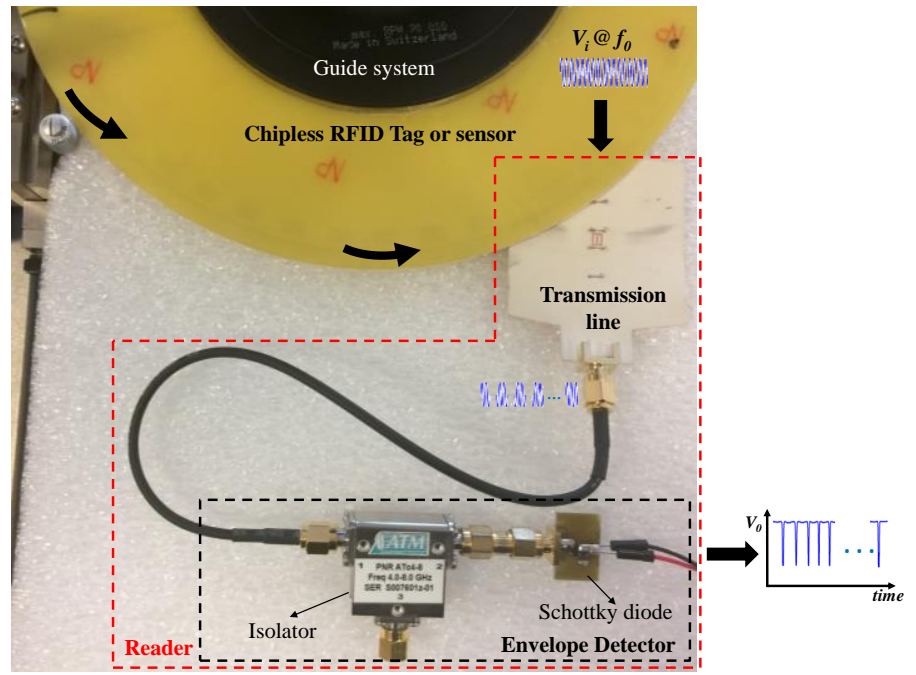

Fig. 8. Photograph of the experimental setup.

(a)

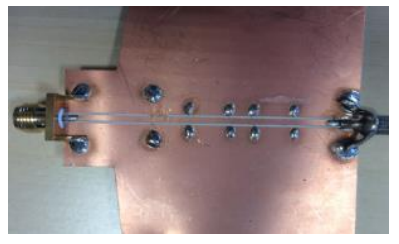

(b)
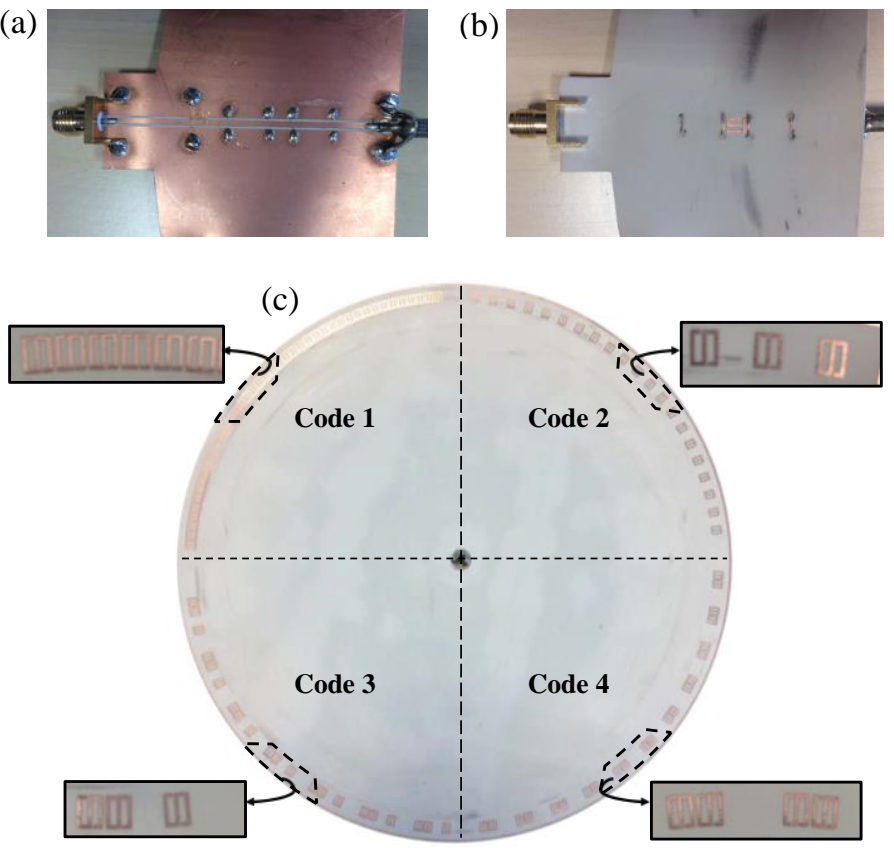

Fig. 9. Photograph of the transmission line reader, including CPW (a) and the S-SRR (b), and the fabricated encoders (c).

However, in the proof-of-concept presented here, circularlyshaped chipless tags are considered. The reason is that by considering circular S-SRR chains, the experimental set-up for angular velocity measurements can be used for tag reading as well. In this paper, four 40-bit circularly-shaped chipless tags have been fabricated and located at the four quadrants of the rotor [Fig. 9(c)]. By injecting a carrier signal with frequency $f_{c}=4 \mathrm{GHz}$ at the input port of the CPW transmission line, it has been possible to obtain the envelope function corresponding to the different 40-bit encoders. The results are depicted in Fig. 10 together with the tag ID codes. The angular velocity of the rotor has been set to $3.5 \mathrm{rpm}$.

It can be appreciated in Fig. 10 that the ID codes of the four 40-bit chipless tags are correctly provided by the proposed reading system. The different notch depths are due to the fact 


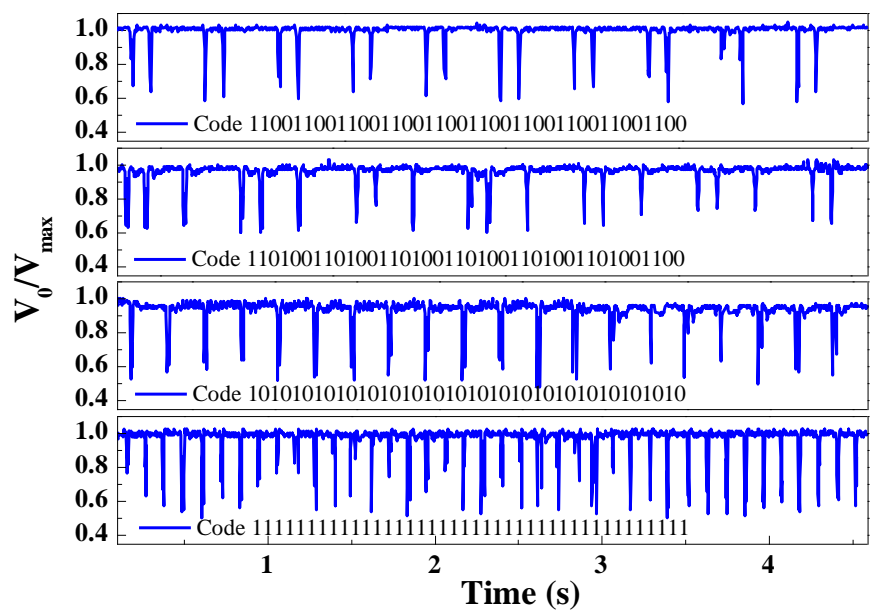

Fig. 10. Measured normalized envelope for four 40-bit fabricated chipless tags with the indicated ID codes.

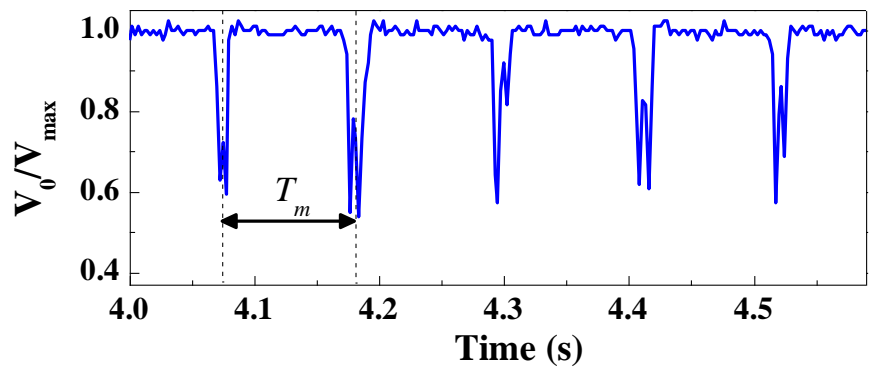

Fig. 11. Measured normalized envelope for a nominal $3.51 \mathrm{rpm}$ speed.

that it is very difficult in practice to ensure a uniform gap separation between the S-SRR of the line and those of the tags (the in-house rotor system experiences some precession movement). Nevertheless, tag reading has been demonstrated and validated. It is worth mentioning that these four 40-bit encoders can be also seen as a single 160-bit chipless RFID tag with ID code composed by the cascade of the individual ID codes. Thus, the number of bits achievable with these approach, based on sequential bit reading by means of a harmonic signal, is only limited by the area occupied by the tag. In the proposed chipless tags, the information capacity per area unit is as high as $7 \mathrm{bit} / \mathrm{cm}^{2}$.

For angular velocity measurements, S-SRRs at all the predefined, and equidistant, positions must be etched (or printed). Note that this is equivalent to a 160-bit encoder with all bits set to the logic state ' 1 '. By using this rotor, quasiinstantaneous velocities can be measured, and changes of angular velocity within a cycle, manifested through variations in the time distance between adjacent notches (or pair of notches), can be detected. Indeed, the proposed system is also useful for angle measurement, and angle resolution is given by the number of resonant elements. For this main reason, and also to be able to detect changes in quasi-instantaneous angular velocities, it is convenient to enhance the number of S-SRR in the rotor as much as possible. However, for constant angular velocity measurements, S-SRR chains with small number of resonators or with absence of resonant elements at certain positions can be used. Indeed, the results given in Fig. 10 are useful to determine the angular velocity of the stepped

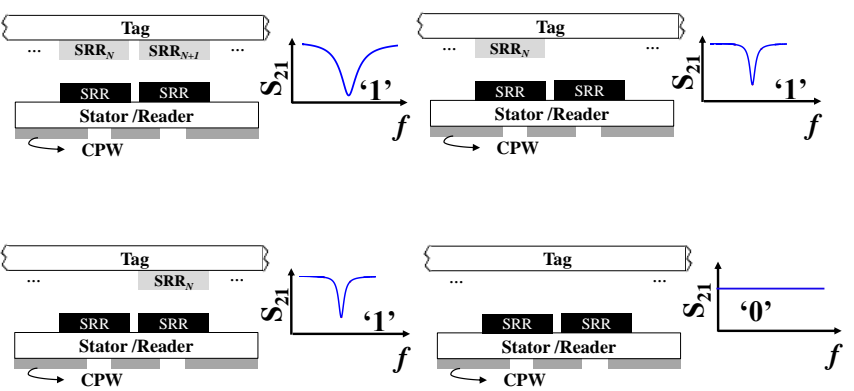

Fig. 12. The four different combinations of SRR pairs (tag) on top of the SRRloaded CPW (reader).

motor (which is constant). Specifically, from the time distance between adjacent notch pairs for the 40-bit encoder with all bits set to ' 1 ' (see details in Fig. 11), the angular velocity is found to be $3.51 \mathrm{rpm}$, i.e., in good agreement with the nominal value (note that the tolerance values of typical step motors are in the vicinity of $1.5 \%$ ).

\section{DISCUSSION}

In [29,30], the reported angular velocity sensor was implemented by means of 300 SRRs etched in the rotor. This was achieved by designing the resonant elements with a rectangular shape. The electrical size of a single S-SRR is not much smaller than the one of the SRR. Therefore, it follows that by using square shaped S-SRRs, it is not possible to accommodate as many resonant elements in the rotor perimeter as in the case of rectangular SRRs (obviously assuming that the resonance frequency is also comparable). In other words, the number of resonant elements per unit length (in the longitudinal direction of the resonator chain) is smaller in this work, as compared to [29,30]. In this regard, rectangular SRRs are a good option to optimize the number of pulses in angular velocity sensors.

However, SRRs are not the optimum resonant elements for chipless RFID tags to be read with SRR-loaded CPW transmission lines as those reported in [29,30], and used as stators. Each logic state is given by a pair of SRRs, rather than one. The reason is that by considering the distance between the slots of the CPW as the period of the SRR chain (in order to maximize the number of resonant elements), it follows that a pair of adjacent SRRs (if they are present in the assigned positions) lie on top of the slots of the CPW transmission line simultaneously. Consequently, a pair of adjacent predefined SRR positions (with presence or absence of resonant elements) cannot be considered as independent bits in chipless RFID encoders based on the SRR chain structure reported in [29,30]. Note that, according to Fig. 12, the four different combinations of SRR pairs (tag) on top of the SRR-loaded CPW (reader) either provide a single notch (a single SRR suffices) or an all-pass response, i.e., two logic states. Taking into account that the area occupied by a pair of rectangular SRRs in the rotor of $[29,30]$ is $23 \mathrm{~mm}^{2}$, and the area of the SSRR used in this work is $9.9 \mathrm{~mm}^{2}$, it follows that the density of information (number of bits per area unit) is more than twice in the present work, as compared to $[29,30]$. 


\section{CONCLUSIONS}

In conclusion, a novel near-field chipless RFID system operating in time domain and based on sequential bit reading has been proposed. The tags are a set of identical resonant elements (S-SRRs in this work) etched (or printed) on a dielectric substrate, and the presence or absence of such elements at predefined positions determines the logic state of the corresponding bit. Tag reading is carried out by near field coupling between a harmonically fed S-SRR-loaded CPW transmission line (reader) and the S-SRRs of the tag, which must be displaced, at constant velocity, above the CPW, in close proximity to it. By this means, the amplitude of the feeding signal is modulated, and the ID code is contained in the envelope function at the output port of the transmission line. Such envelope function can be inferred by means of an envelope detector. In the present paper, four circularly-shaped 40-bit encoders have been proposed, and the ID codes have been inferred by means of an experimental setup consisting of a stepped motor (providing circular motion to the tags) and the necessary electronics (to generate the harmonic carrier signal and to detect the envelope function). Interestingly, the same experimental set-up can be used for angular velocity measurements, where the rotation speed is determined from the time distance between adjacent notches in the envelope function. In this application, it is convenient to implement the S-SRR chain of the rotor with as many resonant elements as possible, since it has direct impact on angle resolution and on the capability to detect rotation speed variations within a cycle. However, in this work, constant angular velocities have been considered and the same encoders used as chipless RFID tags have been found to be suitable for the determination of angular velocities. Chipless RFID tags with unprecedented number of bits have been reported. Indeed, the four 40-bit tags used for validation of the approach can be seen as a single 160-bit tag, which is by far the chipless RFID tag with the largest number of bits reported so far. The information density is also very competitive $\left(7 \mathrm{bit} / \mathrm{cm}^{2}\right)$. The proposed chipless RFID system can be envisaged for applications such as secure paper, where the reading distance can be sacrificed in favor of the number of bits. Specifically, corporate documents, ballots, exams, certificates, etc., with ID codes difficult to copy are some of the potential uses of the proposed chipless tags, providing counterfeiting functionality and a unique identification code, of great interest for security and authentication. In angular velocity sensors, the proposed system, based on S-SRRs, is an alternative to the sensors reported in [29,30], based on pairs of SRRs. In [30], by optimizing the frequency of the feeding signal, 1.200 pulses were achieved. Here, rather than optimizing the number of pulses, the idea was to demonstrate that the encoders can be used for angular velocity measurements. Indeed, for the measurement of constant, or average, rotation speeds, the rotor with four codes can be used for the determination of the rotation direction (clockwise or counterclockwise), provided the sequence of codes is not symmetric in some cases.

\section{REFERENCES}

[1] F. Martin, Artificial Transmission Lines for RF and Microwave Applications, John Wiley, Hoboken, NJ, USA, 2015.

[2] R. Marques, F. Martín, M. Sorolla, Metamaterials with Negative Parameters: Theory, Design and Microwave Applications, John Wiley, Hoboken, NJ, USA, 2007.

[3] F. Martín, F. Falcone, J. Bonache, R. Marqués and M. Sorolla, "Split ring resonator based left handed coplanar waveguide", Appl. Phys. Lett., vol. 83, pp. 4652-4654, Dec. 2003.

[4] F. Falcone, T. Lopetegi, J.D. Baena, R. Marqués, F. Martín and M. Sorolla, "Effective negative- $\varepsilon$ stop-band microstrip lines based on complementary split ring resonators", IEEE Microw. Wireless Compon. Lett., vol. 14, pp. 280-282, Jun. 2004.

[5] J.D. Baena, J. Bonache, F. Martín, R. Marqués, F. Falcone, T. Lopetegi, M.A.G. Laso, J. García, I Gil, M. Flores-Portillo and M. Sorolla, "Equivalent circuit models for split ring resonators and complementary split rings resonators coupled to planar transmission lines", IEEE Trans. Microw. Theory Techn., vol. 53, pp. 1451-1461, Apr. 2005.

[6] J. García-García, F. Martín, F. Falcone, J. Bonache, J.D. Baena, I. Gil, E. Amat, T. Lopetegi, Miguel A.G. Laso, J. A. Marcotegui-Iturmendi, M. Sorolla, and R. Marqués, "Microwave Filters with Improved Stop Band based on Sub-wavelength Resonators", IEEE Trans. Microw. Theory Techn., vol. 53, pp. 1997-2006, Jun. 2005.

[7] F.J. Herraiz-Martínez, G. Zamora, F. Paredes, F. Martín, and J. Bonache, "Multiband printed monopole antennas loaded with open complementary split ring resonators for PANs and WLANs", IEEE Ant. Wireless Propag. Lett., vol. 10, pp. 1528-1531 (2011).

[8] F.J. Herraiz-Martínez, F. Paredes, G. Zamora, F. Martín and J. Bonache, "Dual-band printed dipole antenna loaded with open complementary split-ring resonators (OCSRRs) for wireless applications", Microw. Opt. Technol. Lett., vol. 54, pp. 1014-1017, Apr. 2012.

[9] J. Naqui, M. Durán-Sindreu and F. Martín, "Novel Sensors Based on the Symmetry Properties of Split Ring Resonators (SRRs)", Sensors, vol 11, pp. 7545-7553, 2011.

[10] C. Herrojo, J. Naqui, F. Paredes and F. Martín, "Spectral Signature Barcodes based on S-shaped Split Ring Resonators (S-SRR)", EPJ Applied Metamaterials, vol. 3, pp. 1-6, Jun. 2016.

[11] C. Mandel, B. Kubina, M. Schüßler, and R. Jakoby, "Passive chipless wireless sensor for two-dimensional displacement measurement," Proc. 41st European Microwave Conf., Manchester, UK, 2011, pp. 79-82.

[12] M. Puentes, C. Weiss, M. Schüßler, and R. Jakoby, "Sensor array based on split ring resonators for analysis of organic tissues," IEEE MTT-S Int. Microwave Symp. Dig., Baltimore, MD, June 2011

[13] M. S. Boybay, O. M. Ramahi, "Material characterization using complementary split-ring resonators," IEEE Trans. Instrum. Meas., vol. 61, no. 11, pp. 3039-3046, Nov. 2012.

[14] C.-S. Lee, and C.-L. Yang, "Complementary split-ring resonators for measuring dielectric constants and loss tangents," IEEE Microw. Wireless Compon. Lett., vol. 24, no. 8, pp. 563-565, Aug. 2014.

[15] A. Ebrahimi, W. Withayachumnankul, S. Al-Sarawi, D. Abbott, "Highsensitivity metamaterial-inspired sensor for microfluidic dielectric characterization," IEEE Sensors J. vol. 14, pp. 1345-1351, May 2014.

[16] C.-L. Yang, C.-S. Lee, K.-W. Chen, and K.-Z. Chen, "Noncontact measurement of complex permittivity and thickness by using planar resonators," IEEE Trans. Microw. Theory Techn., vol. 64, no.1, pp. 247-257, Jan. 2016.

[17] L. Su, J. Mata-Contreras, P. Vélez and F. Martín, "Estimation of Conductive Losses in Complementary Split Ring Resonator (CSRR) Loading an Embedded Microstrip Line and Applications", IEEE MTT-S Int. Microw. Symp. (IMS'17), Honolulu, Hawaii, June 2017.

[18] J. Naqui, Symmetry Properties in Transmission Lines Loaded with Electrically Small Resonators: Circuit Modeling and Applications, Springer Theses, 2016.

[19] J. Naqui, M. Durán-Sindreu, F. Martín, "Alignment and position sensors based on split ring resonators", Sensors, vol. 12, Issue 9, pp. 1179011797, September 2012.

[20] A. Karami-Horestani, C. Fumeaux, S.F. Al-Sarawi, and D. Abbott, "Displacement sensor based on diamond-shaped tapered split ring resonator," IEEE Sensors J., vol. 13, no. 4, pp. 1153-1160, Apr. 2013.

[21] A. K. Horestani, J. Naqui, D. Abbott, C. Fumeaux, and F. Martín, "Twodimensional displacement and alignment sensor based on reflection coefficients of open microstrip lines loaded with split ring resonators," Electron Lett., vol. 50, no. 8, pp. 620-622, Apr. 2014. 
[22] J. Naqui, and F. Martín, "Transmission lines loaded with bisymmetric resonators and their application to angular displacement and velocity sensors," IEEE Trans. Microw. Theory Tech., vol. 61, no. 12, pp. 47004713, Dec. 2013.

[23] J. Naqui, and F. Martín, "Angular displacement and velocity sensors based on electric-LC (ELC) loaded microstrip lines", IEEE Sensors J., vol. 14(4), pp. 939-940, April 2014.

[24] J. Naqui, J. Coromina, A. Karami-Horestani, C. Fumeaux, and F. Martín, "Angular displacement and velocity sensors based on coplanar waveguides (CPWs) loaded with S-shaped split ring resonator (S-SRR)", Sensors, vol. 15, pp. 9628-9650, 2015.

[25] J. Naqui, C. Damm, A. Wiens, R. Jakoby, L. Su, and F. Martín, "Transmission lines loaded with pairs of magnetically coupled stepped impedance resonators (SIRs): modeling and application to microwave sensors", IEEE MTT-S Int. Microwave Symp., Tampa, FL, USA, Jun. 2014.

[26] J. Naqui, C. Damm, A. Wiens, R. Jakoby, L. Su, and F. Martín, "Transmission lines loaded with pairs of stepped impedance resonators: modeling and application to differential permittivity measurements", IEEE Trans. Microw. Theory Techn., vol. 64, pp. 3864 - 3877, Nov. 2016.

[27] L. Su, J. Mata-Contreras, J. Naqui, and F. Martín, "Splitter/combiner microstrip sections loaded with pairs of complementary split ring resonators (CSRRs): modeling and optimization for differential sensing applications", IEEE Trans. Microw. Theory Techn., vol. 64(12), pp. 4362-4370, Dec. 2016

[28] L. Su, J. Mata-Contreras, J. Naqui, and F. Martín, "Configurations of splitter/combiner microstrip sections loaded with stepped impedance resonators (SIRs) for sensing applications", Sensors, vol. 16(12), paper 2195, 2016, doi:10.3390/s16122195.

[29] J. Naqui, F. Martín, "Application of broadside-coupled split ring resonator (BC-SRR) loaded transmission lines to the design of rotary encoders for space applications“, IEEE MTT-S Int. Microw. Symp. (IMS'16), San Francisco, May 2016.

[30] J. Mata-Contreras, C. Herrojo, and F. Martín, "Application of split ring resonator (SRR) loaded transmission lines to the design of angular displacement and velocity sensors for space applications", IEEE Trans. Microw. Theory Techn., to be published.

[31] C. S. Hartmann, "A global SAW ID tag with large data capacity," in Proc. of IEEE Ultrasonics Symposium, October 2002, vol. 1, pp. 65-69.

[32] A. Chamarti and K. Varahramyan, "Transmission delay line based ID generation circuit for RFID applications," IEEE Microw. Wireless Compon. Lett., vol. 16, pp. 588-590, 2006.

[33] M. Schüßler, C. Damm, and R. Jakoby, "Periodically LC loaded lines for RFID backscatter applications," in Proc. of Metamaterials 2007, Rome, Italy, October 2007, pp. 103-106

[34] N. Saldanha, D.C. Malocha, "Design Parameters for SAW multi-tone frequency coded reflectors" 2007 IEEE Ultrasonics Symp., pp. 2087 2090, 2007.

[35] M. Schüßler, C. Damm, M. Maasch, and R. Jakoby, "Performance evaluation of left-handed delay lines for RFID backscatter applications," in Proc. of the IEEE MTT-S International Microwave Symposium 2008, pp. $177-180$.

[36] S. Harma, V.P. Plessky, C.S. Hartmann, W. Steichen, "Z-path SAW RFID tag" IEEE Trans. Ultrasonics, Ferroelectric Freq. Control, vol. 55, pp. 208-213, 2008.

[37] H. Tao, W. Weibiao, W. Haodong, S. Yongan, "Reflection and scattering characteristics of reflectors in SAW tags", IEEE Trans. Ultrasonics, Ferroelectric Freq. Control, vol. 55, pp. 1387-1390, 2008.

[38] S. Harma, V.P. Plessky, L. Xianyi, P. Hartogh, "Feasibility of ultrawideband SAW RFID tags meeting FCC rules" IEEE Trans. Ultrasonics, Ferroelectric Freq. Control, vol. 56, pp. 812-820, 2012.

[39] F.J. Herraiz-Martínez, F. Paredes, G. Zamora, F. Martín, and J. Bonache, "Printed magnetoinductive-wave (MIW) delay lines for chipless RFID applications", IEEE Trans. Ant. Propag., vol. 60, pp. 5075-5082, Nov. 2012.

[40] S. Tedjini, E. Perret, A. Vena, D. Kaddout, "Mastering the electromagnetic signature of chipless RFID tags", in Chipless and Conventional Radiofrequency Identification, ed. IGI Global, 2012.

[41] S. Preradovic, I. Balbin, N. C. Karmakar, and G. F. Swiegers, "Multiresonator-based chipless RFID system for low-cost item tracking," IEEE Trans. Microw. Theory Techn., vol. 57, pp. 1411-1419, 2009.

[42] S. Preradovic and N. C. Karmakar, "Design of chipless RFID tag for operation on flexible laminates," IEEE Anten. Wireless Propag. Lett., vol. 9, pp. 207-210, 2010.
[43] O. Rance, R. Siragusa, P. Lemaître-Auger, E. Perret, "Toward RCS magnitude level coding for chipless RFID," IEEE Trans. Microw. Theory Techn., vol. 64, pp. 2315-2325, Jul. 2016.

[44] J. McVay, A. Hoorfar, and N. Engheta, "Space-filling curve RFID tags," in Proc. of 2006 IEEE Radio Wireless Symp., pp. 199-202.

[45] I. Jalaly and D. Robertson, "Capacitively-tuned split microstrip resonators for RFID barcodes," in Proc. of European Microwave Conference, October 2005, vol. 2, pp. 4-7.

[46] H.-S. Jang, W.-G. Lim, K.-S. Oh, S.-M. Moon, and J.-W. Yu, "Design of low-cost chipless system using printable chipless tag with electromagnetic code", IEEE Microw. Wireless Compon. Lett., vol. 20, pp. 640-642, 2010.

[47] A. Vena, E. Perret, and S. Tedjini, "A fully printable chipless RFID tag with detuning correction technique", IEEE Microw. Wireless Compon. Lett., vol. 22(4), pp. 209-211, 2012.

[48] A. Vena, E. Perret, and S. Tedjini, "Design of compact and autocompensated single-layer chipless RFID tag", IEEE Trans. Microw. Theory Techn., vol. 60(9), pp. 2913-2924, Sept. 2012.

[49] A. Vena, E. Perret, and S. Tedjini, "High-capacity chipless RFID tag insensitive to the polarization", IEEE Trans. Ant. Propag., vol. 60(10), pp. 4509-4515, Oct. 2012.

[50] C. Herrojo, J. Naqui, F. Paredes, F. Martín, "Spectral signature barcodes implemented by multi-state multi-resonator circuits for chipless RFID tags", IEEE MTT-S International Microwave Symposium (IMS'16), San Francisco, May 2016.

[51] C. Herrojo, F. Paredes, J. Mata-Contreras, S. Zuffanelli and F. Martín, "Multi-state multi-resonator spectral signature barcodes implemented by means of S-shaped Split Ring Resonators (S-SRR)", IEEE Trans. Microw. Theory Techn., to be published.

[52] M. A. Islam and N. C. Karmakar, "A novel compact printable dualpolarized chipless RFID system," IEEE Trans. Microw. Theory Techn., vol. 60, pp. 2142-2151, Jul. 2012.

[53] A. Vena, E. Perret, S. Tedjini, "Chipless RFID tag using hybrid coding technique," IEEE Trans. Microw. Theory Techn., vol. 59, pp. 3356-3364, Dec. 2011

[54] A. Vena, E. Perret, S. Tedjini, "A compact chipless RFID tag using polarization diversity for encoding and sensing", 2012 IEEE Int. Conf. RFID, pp. 191-197, 2012.

[55] I. Balbin, N.C. Karmakar, "Phase-encoded chipless RFID transponder for large scale low cost applications", IEEE Microw. Wireless. Comp. Lett., vol. 19, pp. 509-511, 2009.

[56] S. Genovesi, F. Costa, A. Monorchio, G. Manara, "Chipless RFID tag exploiting multifrequency delta-phase quantization encoding", IEEE Ant. Wireless Propag. Lett., vol. 15, pp. 738-741, 2015.

[57] H. Chen, L. Ran, J. Huangfu, X. Zhang, K. Chen, T. M. Grzegorczyk, and J. A. Kong, "Left-handed materials composed of only S-shaped resonators," Phys. Rev. E, vol. 70, no. 5, pp. 1-4, Nov. 2004.

[58] H. Chen, L. Ran, J. Huangfu, X. Zhang, K. Chen, T. M. Grzegorczyk, and J. A. Kong, "Negative refraction of a combined double S-shaped metamaterial," Appl. Phys. Lett., vol. 86, no. 15, p. 151909, 2005.

[59] H. Chen, L.-X. Ran, H.-F. Jiang Tao, X.-M. Zhang, K.-S. Cheng, T. M Grzegorczyk, and J. A. Kong, "Magnetic properties of S-shaped split ring resonators," Prog. Electromagn. Res., vol. 51, pp. 231-247, 2005.

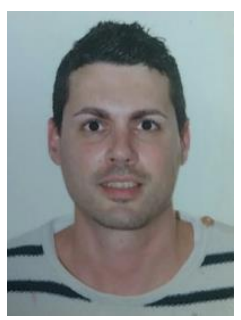

Cristian Herrojo was born in Badalona (Barcelona), Spain, in 1983. He received the Telecommunications Technical Engineering degree (specialty in Electronic Systems) and Telecommunications Engineering degree from Universitat Autònoma de Barcelona, Barcelona (UAB), Spain, in 2010 and 2012, respectively, where, he is currently pursuing the $\mathrm{PhD}$ with a focus on the design of RF/microwave resonant structures applied to RFID tags without chip and he has a research grant from FPI Program of the Education and Science Spanish Ministry.

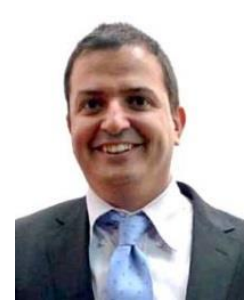

Javier Mata-Contreras was born in 1976 in Málaga (Spain). He received the Ingeniería de Telecomunicación Degree from the Universidad de Málaga (UMA) in 2000 and the $\mathrm{PhD}$ degree from the same university in 2010, with the Thesis "Distributed Amplifiers and Mixers with Transmission Lines based on Metamaterials". In 2000, he joined the UMA Department of Ingeniería de Comunicaciones UMA as 
Assistant Professor. He is currently working at CIMITEC and the Universitat Autònoma de Barcelona as Visitant Professor. His research interests include active and passive microwave devices and active distributed circuits based on metamaterials, among others.

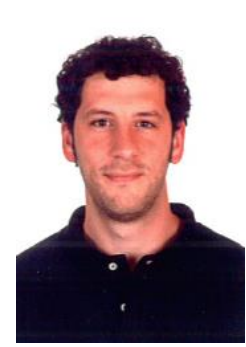

Ferran Paredes was born in Badalona (Barcelona), Spain in 1983. He received the Telecommunications Engineering Diploma (specializing in Electronics) and the Telecommunications Engineering degree from the Universitat Autònoma de Barcelona in 2004 and 2006, respectively and the $\mathrm{PhD}$ degree in Electronics Engineering from the same university in 2012. He was Assistant Professor from 2006 to 2008 at the Universitat Autònoma de Barcelona, where he is currently working as a Research Assistant. His research interests include metamaterial concepts, passive microwaves devices, antennas and RFID.

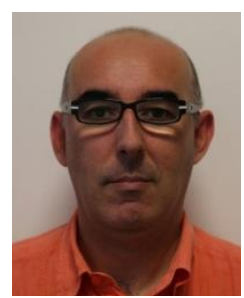

Ferran Martín (M'04-SM'08-F'12) was born in Barakaldo, Spain, in 1965. He received the B.S. degree in physics and Ph.D. degree from the Universitat Autònoma de Barcelona (UAB), Barcelona, Spain, in 1988 and 1992, respectively. From 1994 to 2006 he was Associate Professor in Electronics at the Departament d'Enginyeria Electrònica (Universitat Autònoma de Barcelona), and since 2007 he is Full Professor of Electronics. In recent years, he has been involved in different research activities including modelling and simulation of electron devices for high frequency applications, millimeter wave and $\mathrm{THz}$ generation systems, and the application of electromagnetic bandgaps to microwave and millimeter wave circuits. He is now very active in the field of metamaterials and their application to the miniaturization and optimization of microwave circuits and antennas. He is the head of the Microwave Engineering, Metamaterials and Antennas Group (GEMMA Group) at UAB, and director of CIMITEC, a research Center on Metamaterials supported by TECNIO (Generalitat de Catalunya). $\mathrm{He}$ has organized several international events related to metamaterials, including Workshops at the IEEE International Microwave Symposium (years 2005 and 2007) and European Microwave Conference (2009), and the Fifth International Congress on Advanced Electromagnetic Materials in Microwaves and Optics (Metamaterials 2011), where he has acted as chair of the Local Organizing Committee. He has acted as Guest Editor for three Special Issues on Metamaterials in three International Journals. He has authored and co-authored over 500 technical conference, letter, journal papers and book chapters, he is co-author of the book on Metamaterials entitled Metamaterials with Negative Parameters: Theory, Design and Microwave Applications (John Wiley \& Sons Inc. 2008), author of the book Artificial Transmission Lines for RF and Microwave Applications (John Wiley \& Sons Inc. 2015), and he has generated 16 PhDs. Ferran Martín has filed several patents on metamaterials and has headed several Development Contracts.

Prof. Martín is a member of the IEEE Microwave Theory and Techniques Society (IEEE MTT-S). He is reviewer of the IEEE Transactions on Microwave Theory and Techniques and IEEE Microwave and Wireless Components Letters, among many other journals, and he serves as member of the Editorial Board of IET Microwaves, Antennas and Propagation and International Journal of RF and Microwave Computer-Aided Engineering. He is also a member of the Technical Committees of the European Microwave Conference (EuMC) and International Congress on Advanced Electromagnetic Materials in Microwaves and Optics (Metamaterials). Among his distinctions, Ferran Martín has received the 2006 Duran Farell Prize for Technological Research, he holds the Parc de Recerca UAB - Santander Technology Transfer Chair, and he has been the recipient of two ICREA ACADEMIA Awards (calls 2008 and 2013). He is Fellow of the IEEE since 2012 and Fellow of the IET since 2016. 